\title{
BMJ Open Development and evaluation of percentile distribution of body weight by gestational week as a tool for gestational weight management: a retrospective study based on hospital routine data
}

\author{
Yi Zhang, ${ }^{1}$ Liping Wang, ${ }^{2}$ Chuanmin Yin, ${ }^{3}$ Kai Mu, ${ }^{1}$ Dayan Niu, ${ }^{1}$ Weili Yan $^{1}$
}

To cite: Zhang Y, Wang L, Yin C, et al. Development and evaluation of percentile distribution of body weight by gestational week as a tool for gestational weight management: a retrospective study based on hospital routine data. BMJ Open 2018;8:e019645. doi:10.1136/ bmjopen-2017-019645

- Prepublication history and additional material for this paper are available online. To view these files, please visit the journal online (http://dx.doi. org/10.1136/bmjopen-2017019645).

LW and CY contributed equally.

Received 24 September 2017 Revised 23 March 2018 Accepted 13 April 2018

Check for updates

${ }^{1}$ Department of Clinical Epidemiology, Children's Hospital of Fudan University, Shanghai, China

${ }^{2}$ Maternal and Infant Health Center, International Peace Maternity \& Child Health Hospital of China Welfare Institute, Shanghai, China

${ }^{3}$ Nutrition Department,

Obstetrics and Gynecology

Hospital of Fudan University,

Shanghai, China

Correspondence to

Dr Weili Yan;

yanwl@fudan.edu.cn

\section{ABSTRACT}

Objectives This study aimed to construct and validate smoothed gestational weight centile curves based on preconception weight status for Chinese pregnant women. Design A retrospective study based on hospital routine data

Setting Hospital prenatal care.

Population A cohort of pregnant Chinese women with preconception and gestational body weights without maternal or neonatal complications (sample 1, $\mathrm{n}=2992$ ), and a nonselective independent sample (sample $2, n=7420$ ), were selected from hospital routine data for curve construction and validation.

Study design Smoothed body weight centile curves for each gestational week were constructed using the LMS method in sample 1. Validation in sample 2 included analysis of agreement between predicted weight at the 38th week and observed values using the Bland-Altman Index. Predictions were also compared with international curves.

Results Smoothed centile curves of gestational weight for the three preconception body mass index groups showed a similar non-linear increasing trend. The differences between predicted body weights and observed values were $0.66 \pm 1.58 \mathrm{~kg}, 0.14 \pm 1.61 \mathrm{~kg}$ and $-0.54 \pm 2.06 \mathrm{~kg}$ in the underweight, normal weight and overweight groups, respectively. Bland-Altman Index values were 5.2\%, 5.6\% and $4.7 \%$ in the underweight, normal weight and overweight groups, respectively, with limits of agreement of $-2.4 \sim 3.8$ $\mathrm{kg},-3.0 \sim 3.3 \mathrm{~kg}$ and $-4.4 \sim 3.4 \mathrm{~kg}$, respectively. These limits of agreement were narrower than those of available international curves.

Conclusion Body weight percentiles for gestational weeks 0-42 were proposed for underweight, normal weight or overweight Chinese women. These curves could constitute a useful tool for individualised gestational weight management by predicting body weight at a later gestation phase.

\section{INTRODUCTION}

Promoting healthy gestational weight gain (GWG) is important for preventing obstetric and perinatal morbidity. Low weight gain
Strengths and limitations of this study

- The proposed smoothed percentile curves of gestational weight covered the entire gestation period, from 0 to 42 weeks for the Chinese population by three preconception weight statuses.

- Two independent study samples were used to increase reliability of our findings, one for curve development and the other for validation.

- In the validation analysis, we described how to apply the proposed curves for individualised weight management by computing individualised ideal body weight goals for later gestation weeks based on their weight before or during early gestation.

- The sample size was large in the normal weight group, however, it was relatively limited in the underweight and overweight groups.

during pregnancy is associated with intrauterine growth retardation, low birth weight and future risk of metabolic diseases. ${ }^{1-3}$ Meanwhile, excessive weight gain is related to maternal and fetal complications during pregnancy ${ }^{4-6}$ as well as adverse events later in life, ${ }^{78}$ such as childhood obesity, type 1 diabetes and hypertension. Although recommendations for GWG management are used in antenatal care for monitoring pregnancy weight, the optimal GWG remains controversial. ${ }^{9}$

The recommendations of the Institute of Medicine $(\mathrm{IOM})^{10}$ for gestational weight management in pregnancies with different weight statuses are well known worldwide; however, the reference population mainly included western individuals, with the WHO body mass index (BMI) classification ${ }^{10}$ used for defining the preconception weight status. Studies have suggested that IOM recommendations might not be suitable for Chinese 
pregnancies. ${ }^{11} 12$ One reason is the difference between the Chinese BMI classification $\left(24 \mathrm{~kg} / \mathrm{m}^{2}\right.$ and $28 \mathrm{~kg} / \mathrm{m}^{2}$ for overweight and obesity, respectively) and the preconception weight status in the WHO classification. Another reason is the racial difference in weight gain, as demonstrated in recently published international GWG reference curves constructed by the International Fetal and Newborn Growth Consortium for the 21st Century (INTERGROWTH-21) Project. The latter study found higher GWG in Chinese subjects compared with other racial groups. ${ }^{13}$

Meanwhile, strong associations of early weight gain with adverse pregnancy outcomes have been reported, ${ }^{14} 15$ even with excessive adipose body composition in mid-childhood. ${ }^{16}$ Recommendations for total GWG and weekly rate of weight gain in the second and third trimesters were proposed by IOM; however, recommendations for early gestation were not stressed. ${ }^{10}$ The recent standards generated by the INTERGROWTH-21 project only alert clinicians regarding GWG changes within 14-42 weeks, but not for earlier stage of gestation in normal weight women. The importance of early pregnancy weight management was highlighted ${ }^{13}$ and deserves more attention.

Above all, weight management during pregnancy is of great importance to maternal and child health. However, there are no unified weight management standards during pregnancy for Chinese women, especially describing the optimal weekly rate of weight gain. Therefore, this study used longitudinal data from pregnancies without maternal or fetal complications to generate centile body weight curves, from 0-42 gestational weeks, for Chinese pregnant women in three weight groups with preconception weight status. With such curves, each individual could compute the optimal weight for any gestational week based on current weight, which can be adopted as personal ideal targets for gestational weight management. In addition, the algorithm was validated in a separate sample. We expect the generated curves to serve as a useful tool for individualised weight management during pregnancy.

\section{MATERIALS AND METHODS}

A retrospective cohort of healthy pregnancies was assembled from the electronic medical records of the Obstetrics and Gynecology Hospital of Fudan University, Shanghai, in 2011, to generate the centile curves (derivation cohort).

An independent sample of pregnancies $(n=7420)$ from the International Peace Maternity and Child Health Hospital of the China Welfare Institute (validation cohort) was used to validate the generated centile curves. Clinical data from first antenatal visit to delivery were abstracted. Preconception body weights were not available. No subjects were excluded (pregnancies with adverse maternal or neonatal complications were included).

WHO recommends GWG charts to use longitudinal data in a selected population with low prevalence of maternal and fetal complications, including anthropometric measures before and during pregnancy. ${ }^{17}$ Considering the WHO recommendations and improved representativeness of curves, inclusion criteria in this study comprised Han ethnicity, singleton conception, availability of preconception weight records and healthy pregnancy. Healthy pregnancy was defined by the following criteria: (1) Fasting glucose at first antenatal visit below $5.6 \mathrm{mmol} / \mathrm{L}$. (2) Pregnancy without adverse maternal complications such as pregnancy-induced hypertension or gestational diabetes mellitus (GDM). (3) Pregnancy without neonatal complications, such as fetal growth restriction, fetal distress, low birth weight $(<2500 \mathrm{~g})$, preterm, macrosomia, or Apgar scores below 9 at 1 min or 5 min. The participants' characteristics, perinatal data and neonatal outcomes, including birth weight, height, gestational age and clinical diagnosis, were obtained from the hospital's electronic medical records.

Self-reported preconception weights and heights in obstetric records were used to calculate preconception BMI values. Based on the Chinese BMI classification, ${ }^{18}$ participants were classified into the underweight (preconception $\mathrm{BMI}<18.5 \mathrm{~kg} / \mathrm{m}^{2}$ ), normal weight (preconception $\mathrm{BMI} \geq 18.5 \mathrm{~kg} / \mathrm{m}^{2}$ and $<24 \mathrm{~kg} / \mathrm{m}^{2}$ ) and overweight (preconception BMI $\geq 24 \mathrm{~kg} / \mathrm{m}^{2}$ ) groups. Gestational age was determined by the last menstruation at the time of registration, and corrected by first trimester ultrasonography examination if the difference exceeded 5 days. Serial anthropometric measurements were carried out on calibrated scales at each antenatal care.

Hypertension in pregnancy was defined as diastolic blood pressure over $90 \mathrm{~mm} \mathrm{Hg}$ or systolic blood pressure over $140 \mathrm{~mm} \mathrm{Hg}$ after 20 weeks of pregnancy in women who had a normal blood pressure at the onset of pregnancy. Low birth weight was defined as birth weight under $2500 \mathrm{~g}$, and macrosomia as a birth weight over $4000 \mathrm{~g}$. The mother's weight at term was recorded. GWG was obtained by subtracting the weight at delivery from that recorded preconception, and categorised as insufficient, appropriate and excessive, according to the currently used IOM recommendations for the underweight, normal weight and overweight groups. ${ }^{10}$ GWG represented the difference from first antenatal care to delivery, and was evaluated according to the IOM recommendations in the derivation cohort.

\section{DATA ANALYSIS}

A significance level of $\alpha=0.05$ was used for all tests. Descriptive statistical analysis was performed with Stata V.11 (StataCorp, College Station, Texas, USA). Continuous variables were analysed by $t$-test, and categorical variables by the $\chi^{2}$ test.

Smoothed centile curves of gestational weight by gestational age were generated based on the derivation cohort for the underweight, normal weight and overweight groups by the the lambda, mu, sigma (LMS) method. ${ }^{19}$ The LMS method assumes the Box-Cox power transformation to normalise the data at each age to independent positive values; $\mathrm{L}, \mathrm{M}$ and $\mathrm{S}$ values are cubic splines with knots at each distinct age $(t)$, and were fitted by the maximum penalised likelihood method to create three 
smooth curves: $\mathrm{L}(\mathrm{t})$ Box-Cox power transformation, $\mathrm{M}(\mathrm{t})$ median and $\mathrm{S}(\mathrm{t})$ coefficient of variation. LMSchartmaker (Pro V.1.35, 2006; Cole and Green ${ }^{20}$ ) was employed to create smoothed 5th, 10th, 25th, 50th, 75th, 90th and 95 th centile curves, respectively. We then used the worm plot for residual analysis and determining the goodness of fit, to build a preliminary model. The worm plot shape indicated that the data were similar to the assumed underlying distribution.

Centiles curves at a given gestational week were obtained as:

$$
\begin{aligned}
& \left.\mathrm{C} 100 \alpha(\mathrm{t})=\mathrm{M}(\mathrm{t})(1+\mathrm{L}(\mathrm{t}) \mathrm{S}(\mathrm{t}) \mathrm{Z} \alpha)^{1 / \mathrm{L}(\mathrm{t})} \text { Formula } 1\right) \\
& Z_{\alpha}=\frac{(\mathrm{C} 100 \alpha(\mathrm{t}) / \mathrm{M}(\mathrm{t}))^{L}-1}{L(t) \times S(t)}(\text { Formula } 2)
\end{aligned}
$$

where $\mathrm{Z} \alpha$ is the $\mathrm{SD}$ of the whole sample, and $\mathrm{C} 100 \alpha(\mathrm{t})$ is the corresponding percentile.

The validation cohort was used to assess the constructed percentile curves. First, individual preconception BMI classification was defined based on the observed body weight at early gestation. Z-scores were calculated for body weight at the 16th, 20th and 24th weeks, respectively, based on overall gestational weight curves (online supplementary S-table 1), and their average was defined as $\mathrm{Z}_{1}$-score to compute preconception weight by Formula 1. Each subject was classified into the underweight, normal weight or overweight group based on the estimated preconception weight. Second, the corresponding centile curves and LMS parameters were used to compute body weight for the 38th week. Third, we compared body weights for the 38th week between actual and predicted values by Student's $t$-test. The Bland-Altman plot ${ }^{21}$ was used to examine agreement between actual and predicted weights in the three groups, separately. In the four scatter plots generated, the x-axis represented the mean of paired measurements recorded with two weights, while the y-axis reflected the difference between actual and predicted weights, also known as the bias. The results were interpreted by the Bland-Altman Index,${ }^{21}$ defined as percentage of the difference between predicted and actual weights falling beyond the limit of agreement (LOA). LOA was defined as bias $\pm 1.96 *$ precision, where precision was the SD of the bias. A Bland-Altman Index value of less than $5 \%$ indicated good agreement between actual and predicted weights values. We also performed simple linear correlation analysis to assess consistency.

\begin{tabular}{|c|c|c|c|c|c|}
\hline \multirow[b]{2}{*}{ Characteristics } & \multicolumn{4}{|l|}{ Derivation cohort } & \multirow[b]{2}{*}{ Validation cohort } \\
\hline & $\begin{array}{l}\text { With } \\
\text { preconception } \\
\text { weight }\end{array}$ & $\begin{array}{l}\text { Without } \\
\text { preconception } \\
\text { weight }\end{array}$ & Difference & P values & \\
\hline Age, years, mean & $28.5(2.9)$ & $28.6(3.3)$ & -0.1 & 0.256 & $29.4(3.3)$ \\
\hline Preconception weight, kg mean (SD) & $53.9(7.2)$ & - & - & - & \\
\hline $\begin{array}{l}\text { BMI at the first antenatal visit, } \mathrm{kg} / \mathrm{m}^{2} \text {, } \\
\text { mean (SD) }\end{array}$ & $20.5(2.6)$ & $21.4(2.7)$ & 0.9 & $<0.001$ & $22.5(2.9)$ \\
\hline $\begin{array}{l}\text { Gestational age at the first antenatal } \\
\text { visit, weeks, mean (SD) }\end{array}$ & $17.0(2.2)$ & $16.0(2.1)$ & 1 & $<0.001$ & $24.9(4.5)$ \\
\hline Weight at the first antenatal visit, kg & $57.7(8.0)$ & $56.6(8.0)$ & 1.1 & 0.005 & $59.3(8.4)$ \\
\hline Weight delivery, kg, mean (SD) & 70.7 (8.6) & $70.8(8.7)$ & -0.1 & 0.748 & $70.0(8.9)$ \\
\hline \multicolumn{6}{|l|}{ Gestational weight gain (kg), mean (SD) } \\
\hline$<18.5$ & $12.9(5.0)$ & $14.7(4.6)$ & 1.6 & $<0.001$ & - \\
\hline $18.5-24$ & $13.1(3.9)$ & $14.4(3.9)$ & 1.3 & $<0.001$ & - \\
\hline$\geq 24$ & $11.7(3.7)$ & $12.9(4.2)$ & 1.2 & $<0.001$ & - \\
\hline $\begin{array}{l}\text { GWG exceeded the IOM } \\
\text { recommendations, } \mathrm{n}(\%)\end{array}$ & & & - & - & - \\
\hline$<18.5 \mathrm{~kg} / \mathrm{m}^{2}$ & $67 / 666(10.0)$ & $39 / 245(15.9)$ & - & - & - \\
\hline
\end{tabular}

Table 1 Demographics and characteristics of derivation cohort and validation cohort

GWG obtained by subtracting the body weight at first antenatal visit from the weight at delivery.

BMI, body mass index; GWG, gestational weight gain; IOM, Institute of medicine. 
Recently, the INTERGROWTH-21 project proposed an international GWG reference curve for normal weight women based on data from eight counties, including China. ${ }^{13}$ The preconception BMI of the reference study sample ranged from $18.5 \mathrm{~kg} / \mathrm{m}^{2}$ to $24.9 \mathrm{~kg} / \mathrm{m}^{2}$. To verify the obtained centile distribution curves, a subgroup sample from the validation cohort (with available body weight for the 24th and 38th weeks, and the same preconception BMI) was selected for assessment at the 38 th week for gestational weight, comparing prediction between the INTERGROWTH-21 reference curve and current data by the Bland-Altman method.

\section{Patient and public involvement}

This study is a retrospective data analysis based on routine electronic medical records from two hospitals and was approved by two institutional ethics committees. The extracted data set for analysis did not include patients' personal information. Patients were not involved in the design, recruitment or any measurements of the study. No dissemination of result to patients is planned.

\section{RESULTS}

\section{Description of the derivation cohort}

There were 10685 pregnancies in the study derivation cohort. Of these, 5258 pregnancies with no available preconception weights were excluded; 2420 pregnancies were further excluded for adverse maternal complications; 15 pregnancies with adverse neonatal complications were also excluded. Finally, 2992 healthy pregnancies remained for data analysis, including 666, 2109 and 217 pregnancies in the underweight, normal weight and overweight groups, respectively.

As shown in table 1, selection of a study derivation cohort for constructing gestational weight curves may bring bias. Some characteristics of mothers and neonates in the selected healthy pregnancies with available preconception weights differed from those without preconception weights; these differences were statistically significant but with limited clinical significance. The excluded subjects comprised $20.7 \%$ with GDM, $7.6 \%$ with diagnosed diabetes before gestation, $2.8 \%$ with fasting plasma glucose $\geq 5.1 \mathrm{mmol} / \mathrm{L}$ during pregnancy, $5.3 \%$ with premature newborns, $3.2 \%$ with low birthweight neonates, $6.1 \%$ with macrosomia, $1.8 \%$ with small for gestational age and $10.4 \%$ with fetal distress. GWG from first antenatal care to delivery exceeded the recommended GWG, defined by the IOM recommendations as $10.0 \%, 25.5 \%$ and $44.7 \%$ for underweight, normal weight and overweight individuals, respectively, in the derivation cohort.

\section{Gestational weight centile curves}

Figure 1 depicts centile curves of gestational weight by gestational age (0-42 weeks) for the underweight, normal weight and overweight groups, respectively. The three sets of curves were similar in shape, but different in percentile weight levels. The 5th, 50th and 90th percentiles of GWG from 0 to 42 weeks for the three groups are presented in table 2. Weight gains determined at the 38 th week based on median curves were $15.6 \mathrm{~kg}, 16.1 \mathrm{~kg}$ and $14.6 \mathrm{~kg}$ for the underweight, normal weight and overweight groups, respectively, with highest and lowest values in the normal weight and overweight groups, respectively. In the normal weight group, the 10th, 50 th and 90th percentiles of body weight at the onset of pregnancy were $48.6 \mathrm{~kg}, 54.4 \mathrm{~kg}$ and $61.4 \mathrm{~kg}$, becoming $55.4 \mathrm{~kg}, 62.4 \mathrm{~kg}$ and $70.9 \mathrm{~kg}$ at the 24th week, and $62.5 \mathrm{~kg}$, $70.5 \mathrm{~kg}$ and $79.8 \mathrm{~kg}$ at the $38 \mathrm{th}$ week, respectively. The 5 th, 10th, 25th, 50th, 75th, 90th and 95th percentiles of gestational weight for gestational weeks 0 to 42 are shown in online supplementary S-table 2-4; L, M and $\mathrm{S}$ parameters by gestation week (0 to 42 ) are presented for the underweight, normal weight and overweight groups, as well as all subjects in online supplementary S-table 1.

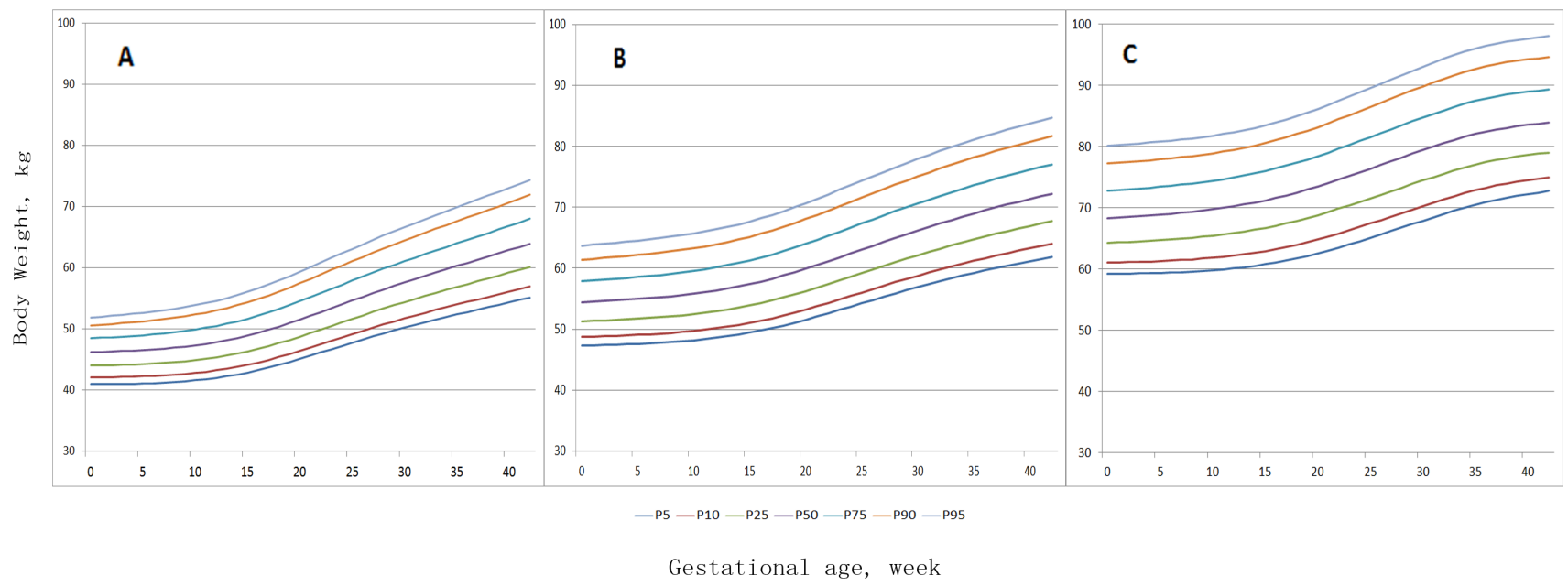

Figure 1 Gestational weight centile curves for underweight (A), normal weight (B) and overweight (C) pregnancies. 
Table 2 Percentiles of gestational weight gain (GWG) by gestational age (week) for pregnant women with different preconception weight status

\begin{tabular}{|c|c|c|c|c|c|c|c|c|c|}
\hline \multirow{3}{*}{$\begin{array}{l}\text { Gestation age, } \\
\text { week }\end{array}$} & \multicolumn{3}{|c|}{ Underweight(kg) } & \multicolumn{3}{|c|}{ Normal weight(kg) } & \multicolumn{3}{|c|}{ Overweight (kg) } \\
\hline & \multicolumn{3}{|c|}{$\mathrm{BMI}<18.5 \mathrm{~kg} / \mathrm{m}^{2}$} & \multicolumn{3}{|c|}{$\mathrm{BMI}=18.5 \mathrm{~kg} / \mathrm{m}^{2}-23.9 \mathrm{~kg} / \mathrm{m}^{2}$} & \multicolumn{3}{|c|}{$\mathrm{BMI} \geq 24 \mathrm{~kg} / \mathrm{m}^{2}$} \\
\hline & P5 & P50 & P95 & P5 & P50 & P95 & P5 & P50 & P95 \\
\hline 0 & 40.9 & 46.2 & 51.9 & 47.3 & 54.4 & 63.7 & 59.0 & 69.3 & 84.7 \\
\hline 1 & 41.0 & 46.2 & 52.0 & 47.3 & 54.5 & 63.8 & 59.2 & 69.5 & 85.0 \\
\hline 2 & 41.0 & 46.3 & 52.1 & 47.4 & 54.6 & 64.0 & 59.4 & 69.8 & 85.4 \\
\hline 3 & 41.0 & 46.4 & 52.3 & 47.4 & 54.7 & 64.2 & 59.6 & 70.0 & 85.7 \\
\hline 4 & 41.0 & 46.5 & 52.5 & 47.5 & 54.8 & 64.3 & 59.7 & 70.3 & 86.0 \\
\hline 5 & 41.1 & 46.5 & 52.6 & 47.6 & 55.0 & 64.5 & 59.9 & 70.5 & 86.3 \\
\hline 6 & 41.1 & 46.6 & 52.8 & 47.7 & 55.1 & 64.7 & 60.1 & 70.8 & 86.6 \\
\hline 7 & 41.2 & 46.8 & 53.0 & 47.8 & 55.2 & 64.9 & 60.2 & 71.0 & 86.9 \\
\hline 8 & 41.3 & 46.9 & 53.3 & 47.9 & 55.4 & 65.2 & 60.4 & 71.3 & 87.2 \\
\hline 9 & 41.4 & 47.1 & 53.6 & 48.0 & 55.6 & 65.4 & 60.6 & 71.5 & 87.4 \\
\hline 10 & 41.6 & 47.3 & 53.9 & 48.2 & 55.8 & 65.7 & 60.8 & 71.8 & 87.7 \\
\hline 11 & 41.8 & 47.5 & 54.2 & 48.4 & 56.1 & 66.0 & 60.9 & 72.0 & 87.9 \\
\hline 12 & 42.0 & 47.8 & 54.6 & 48.6 & 56.3 & 66.4 & 61.1 & 72.2 & 88.2 \\
\hline 13 & 42.3 & 48.1 & 55.0 & 48.8 & 56.7 & 66.8 & 61.3 & 72.5 & 88.4 \\
\hline 14 & 42.5 & 48.5 & 55.5 & 49.1 & 57.0 & 67.2 & 61.5 & 72.7 & 88.7 \\
\hline 15 & 42.9 & 48.9 & 56.0 & 49.4 & 57.4 & 67.7 & 61.7 & 73.0 & 88.9 \\
\hline 16 & 43.3 & 49.4 & 56.6 & 49.8 & 57.8 & 68.2 & 61.9 & 73.3 & 89.2 \\
\hline 17 & 43.7 & 49.8 & 57.2 & 50.2 & 58.3 & 68.8 & 62.2 & 73.6 & 89.5 \\
\hline 18 & 44.1 & 50.4 & 57.9 & 50.6 & 58.8 & 69.4 & 62.5 & 74.0 & 89.8 \\
\hline 19 & 44.6 & 50.9 & 58.6 & 51.1 & 59.3 & 70.0 & 62.8 & 74.4 & 90.2 \\
\hline 20 & 45.1 & 51.5 & 59.3 & 51.6 & 59.9 & 70.7 & 63.2 & 74.8 & 90.6 \\
\hline 21 & 45.6 & 52.1 & 60.1 & 52.1 & 60.5 & 71.4 & 63.6 & 75.3 & 91.1 \\
\hline 22 & 46.1 & 52.8 & 60.8 & 52.6 & 61.1 & 72.1 & 64.0 & 75.8 & 91.6 \\
\hline 23 & 46.7 & 53.4 & 61.6 & 53.1 & 61.8 & 72.9 & 64.5 & 76.3 & 92.1 \\
\hline 24 & 47.2 & 54.0 & 62.3 & 53.7 & 62.4 & 73.6 & 65.0 & 76.9 & 92.7 \\
\hline 25 & 47.7 & 54.6 & 63.1 & 54.2 & 63.1 & 74.4 & 65.5 & 77.5 & 93.3 \\
\hline 26 & 48.3 & 55.3 & 63.8 & 54.8 & 63.7 & 75.1 & 66.1 & 78.0 & 93.8 \\
\hline 27 & 48.8 & 55.9 & 64.6 & 55.3 & 64.4 & 75.8 & 66.6 & 78.6 & 94.4 \\
\hline 28 & 49.3 & 56.4 & 65.3 & 55.9 & 65.0 & 76.6 & 67.1 & 79.1 & 95.0 \\
\hline 29 & 49.8 & 57.0 & 66.0 & 56.4 & 65.6 & 77.3 & 67.6 & 79.6 & 95.5 \\
\hline 30 & 50.2 & 57.6 & 66.6 & 56.9 & 66.2 & 77.9 & 68.1 & 80.2 & 96.0 \\
\hline 31 & 50.7 & 58.1 & 67.3 & 57.4 & 66.8 & 78.6 & 68.6 & 80.7 & 96.5 \\
\hline 32 & 51.1 & 58.7 & 68.0 & 57.9 & 67.4 & 79.3 & 69.1 & 81.2 & 97.1 \\
\hline 33 & 51.5 & 59.2 & 68.6 & 58.4 & 67.9 & 79.9 & 69.6 & 81.7 & 97.6 \\
\hline 34 & 51.9 & 59.7 & 69.2 & 58.8 & 68.5 & 80.5 & 70.1 & 82.2 & 98.1 \\
\hline 35 & 52.4 & 60.3 & 69.9 & 59.3 & 69.0 & 81.1 & 70.6 & 82.6 & 98.5 \\
\hline 36 & 52.8 & 60.8 & 70.5 & 59.7 & 69.5 & 81.7 & 71.1 & 83.1 & 99.0 \\
\hline 37 & 53.2 & 61.3 & 71.2 & 60.1 & 70.0 & 82.2 & 71.5 & 83.5 & 99.4 \\
\hline 38 & 53.6 & 61.8 & 71.8 & 60.5 & 70.5 & 82.8 & 71.9 & 83.9 & 99.7 \\
\hline 39 & 54.0 & 62.4 & 72.4 & 60.8 & 70.9 & 83.3 & 72.3 & 84.2 & 100.0 \\
\hline 40 & 54.3 & 62.9 & 73.1 & 61.2 & 71.4 & 83.8 & 72.6 & 84.5 & 100.3 \\
\hline 41 & 54.7 & 63.4 & 73.7 & 61.5 & 71.8 & 84.2 & 73.0 & 84.8 & 100.6 \\
\hline
\end{tabular}


Table 2 Continued

\begin{tabular}{|c|c|c|c|c|c|c|c|c|c|}
\hline \multirow{3}{*}{$\begin{array}{l}\text { Gestation age, } \\
\text { week }\end{array}$} & \multicolumn{3}{|c|}{ Underweight(kg) } & \multicolumn{3}{|c|}{ Normal weight(kg) } & \multicolumn{3}{|c|}{ Overweight (kg) } \\
\hline & \multicolumn{3}{|c|}{$\mathrm{BMI}<18.5 \mathrm{~kg} / \mathrm{m}^{2}$} & \multicolumn{3}{|c|}{$\mathrm{BMI}=18.5 \mathrm{~kg} / \mathrm{m}^{2}-23.9 \mathrm{~kg} / \mathrm{m}^{2}$} & \multicolumn{3}{|c|}{$\mathrm{BMI} \geq 24 \mathrm{~kg} / \mathrm{m}^{2}$} \\
\hline & P5 & P50 & P95 & P5 & P50 & P95 & P5 & P50 & P95 \\
\hline 42 & 55.1 & 63.9 & 74.4 & 61.9 & 72.2 & 84.7 & 73.3 & 85.1 & 100.8 \\
\hline GWG (0-42), kg & 14.2 & 17.7 & 22.5 & 14.6 & 17.8 & 21.0 & 14.3 & 15.9 & 16.1 \\
\hline GWG (0-40), kg & 13.4 & 16.7 & 21.2 & 13.9 & 17.0 & 20.1 & 13.6 & 15.2 & 15.6 \\
\hline GWG(0-38), kg & 12.6 & 15.6 & 19.9 & 13.2 & 16.1 & 19.1 & 12.9 & 14.6 & 15.0 \\
\hline
\end{tabular}

\section{Validation of the curves}

Overall, the predicted weights were close to the actual ones observed. The differences were reduced in the underweight and normal weight groups compared with the overweight group $(0.66 \pm 1.58 \mathrm{~kg}, 0.14 \pm 1.61 \mathrm{~kg}$ and $-0.54 \pm 2.06 \mathrm{~kg}$, respectively; all $\mathrm{p}<0.001)$. The actual weights were well correlated with the predicted values in all three groups (correlation coefficients of 0.92, 0.96 and 0.95 for underweight, normal weight and overweight individuals, respectively; all $\mathrm{p}<0.001)$. Bland-Altman Index values were $5.2 \%, 5.6 \%$ and $4.7 \%$, respectively, for the underweight, normal weight and overweight groups, indicating that nearly $5 \%$ of subjects had gestational weights beyond $95 \%$ CIs of predicted weights at the 38th week (figure 2 and online supplementary S-table 1).

\section{Comparison with the INTERGROWTH-21 gestational weight curve}

A subgroup sample with normal preconception body weights $\left(\mathrm{n}=2302\right.$, preconception $\mathrm{BMI}=18.5 \mathrm{~kg} / \mathrm{m}^{2}-$ $24.99 \mathrm{~kg} / \mathrm{m}^{2}$ ) from the validation cohort was selected for comparison. The actual body weight at 38 th week was $70.7 \pm 6.3 \mathrm{~kg}$. Meanwhile, the predicted body weights based on centile curves proposed by the current study and INTERGROWTH-21 ${ }^{13}$ were very similar $(70.7 \pm 6.1 \mathrm{~kg}$ and $69.7 \pm 7.1 \mathrm{~kg}$, respectively). Bland-Altman Index values were $4.7 \%$ and $4.0 \%$, respectively; however, narrower limits of agreement were obtained from the current study (LOA current study),$-3.9 \sim 3.9$ vs $\left.\operatorname{LOA}_{\text {INTERGROWTH-2l }},-7 \sim 5\right)$ (figure 3).

\section{DISCUSSION}

Based on longitudinal body weight records of a group of healthy pregnancies without maternal or neonatal complications during gestation as the derivation cohort, we constructed the first gestational weight centile curves of the three preconception BMI categories over the entire pregnancy in Chinese women. The proposed gestational weight centile curves were validated in an independent sample and showed good agreement between predicted and actual body weights for an upcoming gestational week, especially in pregnancies with normal preconception BMIs. The performance in predicting the body weight for a later gestational week was also improved compared with using newly published international curves.

Maternal GWG management is a key component of prenatal care. A centile curve based on pregnant Chinese women may be helpful for weight management in the
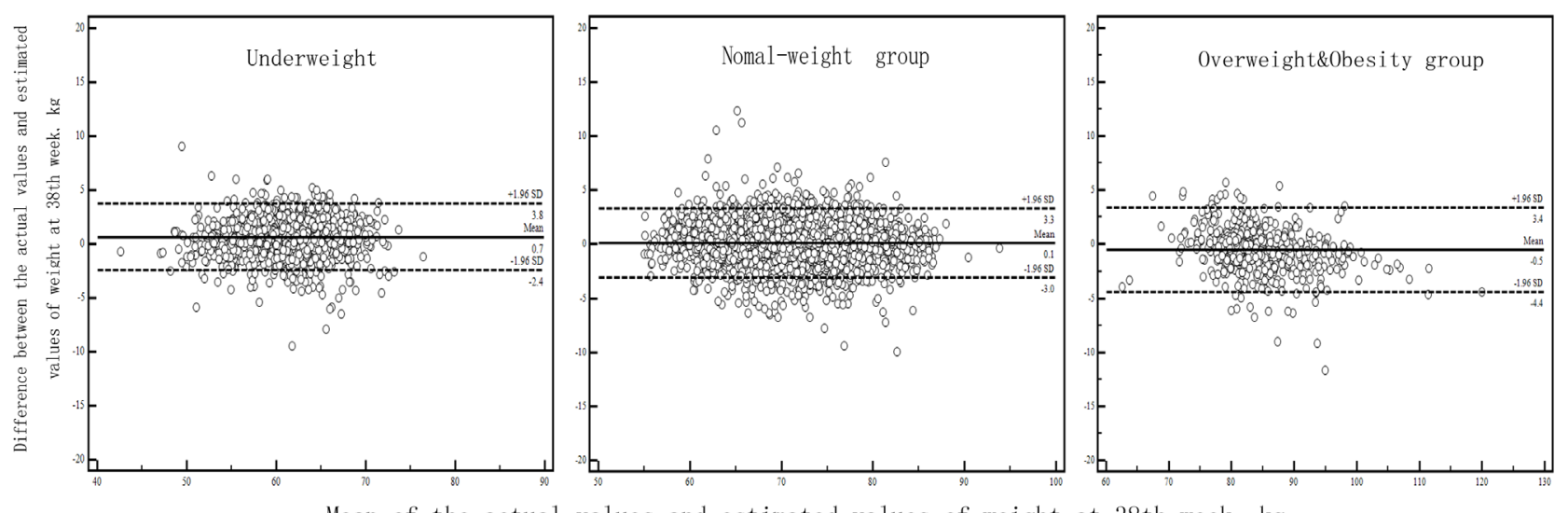

Mean of the actual values and estimated values of weight at 38 th week, $k g$

$$
\begin{aligned}
& \text { - Mean of difference between the actual values and estimated val ues of weight at } 38^{\mathrm{h}} \text { week } \\
& \text { Upper and low agreement limits }(+/-1.96 \mathrm{SD})
\end{aligned}
$$

Figure 2 Bland-Altman plot assessing the agreement between the actual values and predicted values of body weight at the 38th week for three groups of pregnancies with different preconception weight status. 


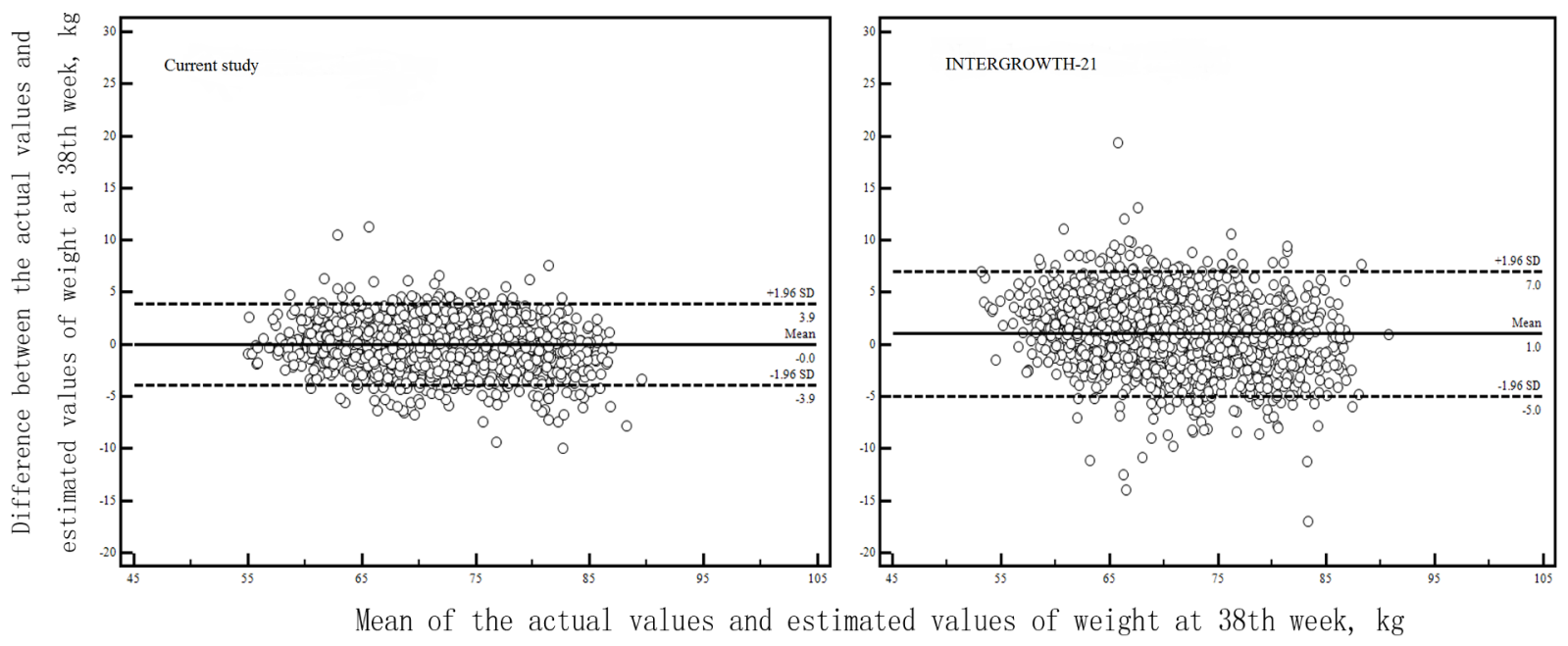

Mean of difference between the actual values and estimated values of weight at $38^{\text {th }}$ week -...-...- Upper and low agreement limits $(+/-1.96 \mathrm{SD})$

Figure 3 Bland-Altman plot assessing the agreement between actual values and predicted values of body weight at the 38th week by using reference curves of gestational weight gain (GWG) proposed by the current study and INTERGROWTH-21 $(n=2302$ from the validation cohort).

pregnant population of China. Recommendations for GWG were first established by IOM in 1990 and amended in 2009 for the three pregnant weight statuses, when faced with the increasing prevalence of obesity, elevated age of pregnant women and new knowledge about pregnancy. ${ }^{1022} 23$ Studies using the IOM recommendations in Chinese pregnancies demonstrated that excessive weight gain in pregnant Chinese women is a serious problem. ${ }^{24}{ }^{25}$ In the INTERGROWTH-21 project, healthy pregnant Chinese women (accounting for $10 \%$ of the sample size) showed significantly higher GWG compared with other ethnic groups. ${ }^{13}$ Therefore, gestational weight management for Chinese women needs race-specific recommendations that may allow for greater GWG.

The current study proposed smoothed centile curves of gestational weight for pregnant Chinese women with three merits. First, three sets of centile curves provided an optimal weight for any future gestational week based on the individual's current or preconception weight, specifically for underweight, overweight and obese pregnancies, who are not included in recent international GWG curves. ${ }^{13}$ Second, the three sets of curves covering early stage of gestation, make individualised weight gain recommendations for early gestation possible. Eun-Hee Cho found that excessive weight gain in early pregnancy is a significant risk factor for multiple adverse pregnancy outcomes among Korean women, who are similar to Chinese women in terms of racial background. ${ }^{26}$ Other studies have reported that excessive weight gain in early pregnancy increases the incidence of impaired glucose tolerance ${ }^{27}$ and GDM, ${ }^{28}$ as well as the birth weight of the offspring. ${ }^{29}$ The three curves recommended a weekly rate of GWG, at weeks 0 to 42, and could help manage gestational weight in the entire pregnancy period. Finally, the algorithm generated based on curves was able to predict the weight at any given gestational week accurately. The difference between predicted and actual weights was smallest for normal weight pregnancies, followed by the underweight and overweight/obese groups. By selecting a subsample from the validation cohort in the current study with the same BMI range as INTERGROWTH-21 subjects, predicted body weights at the 38th week obtained by curves from this study were consistent with the actual weights, but with increased precision compared with INTERGROWTH-21 references. Compared with two previous studies based on Singaporean ${ }^{11}$ and Chinese ${ }^{12}$ populations, the current study was superior in methodology.

Gynaecologists, obstetricians and pregnant women undertaking individualised weight management can apply the gestational weight centile curves proposed in this study. Based on the preconception weight status or the weight during early gestation, the proposed algorithm could help individual pregnant women estimate the recommended body weight for subsequent gestational weeks.

Several limitations should be considered in the interpretation of these results. First, preconception weights and heights were self-reported by patients, indicating a possible recall bias. Second, selection criteria for healthy pregnancy were based on characteristics from clinical records, and blood indicators such as glycosylated haemoglobin and insulin, were not available. Third, the sample size was limited, especially for the underweight and overweight groups. Fourth, the representativeness of the current study is limited since the derivation cohort was from a single hospital. Future perspective studies with larger sample sizes and broader representativeness are expected to improve the proposed curves. 


\section{CONCLUSIONS}

We proposed and validated the first gestational weight centile curves by gestational week for pregnant Chinese women with different preconception weight statuses. The generated curves could serve as a useful tool for individualised gestational weight management. The curves for women with normal weights before pregnancy yielded more accurate predictability for subsequent gestational weeks; those of underweight and overweight pregnant women need improvement in future studies with larger sample sizes.

Acknowledgements The authors thank MedSci (contract No. 1712262545-A) for the English language editing service.

Contributors WY had full access to all the data in the study and takes responsibility for the integrity of the data and the accuracy of the data analysis. Study concept and design: WY. Acquisition, analysis or interpretation of data: LW, CY, YZ, WY. Drafting of the manuscript: YZ, WY. Critical revision of the manuscript for important intellectual content: YZ, LW, CY, KM, DN, WY. Statistical analysis: YZ, WY. Obtained funding: WY, YZ. Administrative, technical or material support: LW, CY, KM, DN. Study supervision: WY. All authors read and approved the final version of the manuscript.

Funding This work is supported by Natural Science Foundation of China (Grant No: 81273168); National key research and development program (Grant No: 2016YFC1000500); Shanghai Health and Family Planning Commission of Outstanding Academic Leaders Plan (Grant No: XBR2013101); and Intramural grant of Children's Hospital of Fudan University.

Competing interests None declared.

Patient consent Not required.

Ethics approval Ethics Committee of Obstetrics and Gynecology Hospital of Fudan University and Institutional Review Board of International Peace Maternity and Child Health Hospital, Shanghai, China.

Provenance and peer review Not commissioned; externally peer reviewed.

Data sharing statement Data is available upon request from the corresponding author (WY).

Open access This is an open access article distributed in accordance with the Creative Commons Attribution Non Commercial (CC BY-NC 4.0) license, which permits others to distribute, remix, adapt, build upon this work non-commercially, and license their derivative works on different terms, provided the original work is properly cited, appropriate credit is given, any changes made indicated, and the use is non-commercial. See:Chttp://creativecommons.org/licenses/by-nc/4.0/.

(c) Article author(s) (or their employer(s) unless otherwise stated in the text of the article) 2018. All rights reserved. No commercial use is permitted unless otherwise expressly granted.

\section{REFERENCES}

1. Wen T, Lv Y. Inadequate gestational weight gain and adverse pregnancy outcomes among normal weight women in China. Int $J$ Clin Exp Med 2015;8:2881-6.

2. Hulsey TC, Neal D, Bondo SC, et al. Maternal prepregnant body mass index and weight gain related to low birth weight in South Carolina. South Med J 2005;98:411-5.

3. Walker LO, Hoke MM, Brown A. Risk factors for excessive or inadequate gestational weight gain among Hispanic women in a U.S.-Mexico border state. J Obstet Gynecol Neonatal Nurs 2009;38:418-29.

4. Chung JG, Taylor RS, Thompson JM, et al. Gestational weight gain and adverse pregnancy outcomes in a nulliparous cohort. Eur $J$ Obstet Gynecol Reprod Biol 2013;167:149-53.
5. Einerson BD, Huffman JK, Istwan NB, et al. New gestational weight gain guidelines: an observational study of pregnancy outcomes in obese women. Obesity 2011;19-2361-4.

6. Gaillard R, Durmus B, Hofman A, et al. Risk factors and outcomes of maternal obesity and excessive weight gain during pregnancy. Obesity 2013;21-1046-55.

7. Ensenauer R, Chmitorz A, Riedel C, et al. Effects of suboptimal or excessive gestational weight gain on childhood overweight and abdominal adiposity: results from a retrospective cohort study. Int $J$ Obes 2013;37:505-12.

8. Poston L. Maternal obesity, gestational weight gain and diet as determinants of offspring long term health. Best Pract Res Clin Endocrinol Metab 2012;26:627-39.

9. Abrams B, Altman SL, Pickett KE. Pregnancy weight gain: still controversial. Am J Clin Nutr 2000;71(Suppl):1233S-41.

10. Rasmussen KM, Yaktine AL. Weight gain during pregnancy: reexamining the guidelines. Washington (DC): National Academies Press, 2009.

11. Ee TX, Allen JC, Malhotra R, et al. Determining optimal gestational weight gain in a multiethnic Asian population. J Obstet Gynaecol Res 2014;40:1002-8.

12. Yang S, Peng A, Wei S, et al. Pre-pregnancy body mass index, gestational weight gain, and birth weight: a cohort study in China. PLoS One 2015;10:e0130101.

13. Cheikh Ismail L, Bishop DC, Pang R, et al. Gestational weight gain standards based on women enrolled in the fetal growth longitudinal study of the INTERGROWTH-21st project: a prospective longitudinal cohort study. BMJ 2016;352:i555.

14. Brunner S, Stecher L, Ziebarth S, et al. Excessive gestational weight gain prior to glucose screening and the risk of gestational diabetes: a meta-analysis. Diabetologia 2015;58:2229-37.

15. Cho EH, Hur J, Lee KJ. Early gestational weight gain rate and adverse pregnancy outcomes in Korean women. PLoS One 2015;10:e0140376.

16. Hivert MF, Rifas-Shiman SL, Gillman MW, et al. Greater early and mid-pregnancy gestational weight gains are associated with excess adiposity in mid-childhood. Obesity 2016;24:1546-53.

17. Zaykin D, Zhivotovsky L, Weir BS. Exact tests for association between alleles at arbitrary numbers of loci. Genetica 1995;96:169-78.

18. Wang $\mathrm{Y}, \mathrm{Mi} \mathrm{J}$, Shan $\mathrm{XY}$, et al. Is China facing an obesity epidemic and the consequences? The trends in obesity and chronic disease in China. Int J Obes 2007;31-177-88.

19. Cole TJ. The LMS method for constructing normalized growth standards. Eur J Clin Nutr 1990;44:45-60.

20. Cole TJ, Green PJ. Smoothing reference centile curves: the LMS method and penalized likelihood. Stat Med 1992;11:1305-19.

21. Bland JM, Altman DG. Statistical methods for assessing agreement between two methods of clinical measurement. Lancet 1986;1:307-10.

22. Flegal KM, Carroll MD, Kuczmarski RJ, et al. Overweight and obesity in the United States: prevalence and trends, 1960-1994. Int J Obes Relat Metab Disord 1998;22:39-47.

23. Yoon $\mathrm{KH}$, Lee JH, Kim JW, et al. Epidemic obesity and type 2 diabetes in Asia. Lancet 2006;368:1681-8.

24. Yang YD, Yang HX. [Investigation into the clinical suitability of Institute of Medicine 2009 guidelines regarding weight gain during pregnancy for women with full term singleton fetus in China]. Zhonghua Fu Chan Ke Za Zhi 2012;47:646-50.

25. Wei YM, Yang HX, Gao XL. [Investigation into the prevalence and suitable diagnostic criteria of gestational diabetes mellitus in China]. Zhonghua Fu Chan Ke Za Zhi 2008;43:647-50.

26. Cho EH, Hur J, Lee KJ. Early gestational weight gain rate and adverse pregnancy outcomes in korean women. PLoS One 2015;10:e0140376.

27. Herring SJ, Oken E, Rifas-Shiman SL, et al. Weight gain in pregnancy and risk of maternal hyperglycemia. Am J Obstet Gynecol 2009;201:61.e1-61.e7.

28. Hedderson MM, Gunderson EP, Ferrara A. Gestational weight gain and risk of gestational diabetes mellitus. Obstet Gynecol 2010;115:597-604.

29. Brown JE, Murtaugh MA, Jacobs DR, et al. Variation in newborn size according to pregnancy weight change by trimester. Am J Clin Nutr 2002;76:205-9. 